\title{
Theoretical Studies of the Spin Hamiltonian Parameters and Local Structures for the Two Tetragonal $\mathrm{Cu}^{2+}$ Centers in $\mathrm{Ca}(\mathrm{OH})_{2}$
}

\author{
H.M. ZHANG ${ }^{a, b, *}$, S.Y. WU ${ }^{a, c}$ AND Z.H. ZHANG ${ }^{a}$ \\ ${ }^{a}$ Department of Applied Physics, University of Electronic Science and Technology of China \\ Chengdu 610054, P.R. China \\ ${ }^{b}$ Key Laboratory of Nondestructive Test, Ministry of Education \\ Nanchang Hangkong University, Nanchang 330063, P.R. China \\ ${ }^{c}$ International Centre for Materials Physics, Chinese Academy of Sciences, Shenyang 110016, P.R. China
}

(Received October 30, 2010)

\begin{abstract}
The spin Hamiltonian parameters ( $g$ factors $g_{\|}, g_{\perp}$ and the hyperfine structure constants $A_{\|}, A_{\perp}$ ) and the local structures for the two tetragonal $\mathrm{Cu}^{2+}$ centers I and II in $\mathrm{Ca}(\mathrm{OH})_{2}$ are theoretically studied from the perturbation formulae of these parameters for a $3 d^{9}$ ion under tetragonally elongated octahedra. The $\left[\mathrm{Cu}(\mathrm{OH})_{6}\right]^{4-}$ clusters on the substitutional $\mathrm{Ca}^{2+}$ site are found to suffer the relative elongations by about $0.083 \AA$ and $0.065 \AA$ for centers I and II, respectively, along the $C_{4}$ axis due to the Jahn-Teller effect. The above tetragonal elongations may entirely depress the original trigonal distortion of the host $\mathrm{Ca}^{2+}$ site in $\mathrm{Ca}(\mathrm{OH})_{2}$. The calculated spin Hamiltonian parameters based on the above Jahn-Teller elongations show good agreement with the experimental results. The EPR spectra and the local structures for the two centers are compared with one another.
\end{abstract}

PACS: $76.30 . \mathrm{Fc}$

\section{Introduction}

Calcium dihydroxide $\left(\mathrm{Ca}(\mathrm{OH})_{2}\right)$ crystals have attracted extensive interest of researchers due to the unique structural and vibrational $[1,2]$ and high pressure $[3,4]$ properties as well as antimicrobial activity $[5,6]$. In particular, novel local structures and electronic energy levels can be produced in these materials doped with some transition-metal ions (e.g., $\mathrm{Fe}^{3+}, \mathrm{Mn}^{2+}, \mathrm{Co}^{2+}$ ), which may be conveniently investigated by means of the electron paramagnetic resonance (EPR) technique [7-9]. Among the transition-metal ions, $\mathrm{Cu}^{2+}$ of the $3 d^{9}$ configuration (equivalent to one $3 d$ hole) is a model system with only one ground state and one excited state under ideal octahedral crystal fields, and the EPR analysis for $\mathrm{Cu}^{2+}$ in crystals can provide useful information about the local structures and electronic states of the dopants in the hosts [10-12]. The EPR experiments were carried out for $\mathrm{Cu}^{2+}$ doped $\mathrm{Ca}(\mathrm{OH})_{2}$, and the spin Hamiltonian (SH) parameters (the $g$ factors $g_{\|}, g_{\perp}$ and the hyperfine structure constants $A_{\|}, A_{\perp}$ ) were also measured for the two tetragonal $\mathrm{Cu}^{2+}$ centers I and II at $87 \mathrm{~K}$ and room temperature, respectively [13].

In order to explain the experimental EPR results, the

* corresponding author; e-mail: huamingzhang66@gmail.com previous work [13] adopted the simple second-order perturbation formulae of the $\mathrm{SH}$ parameters with various adjustable parameters (e.g., $u$ and $w$ ), in view of the JahnTeller effect. However, there are some imperfections in their treatments. First, the calculations of the SH parameters were not correlated with the local structures of the $\mathrm{Cu}^{2+}$ centers but treated by fitting the experimental data. Meanwhile, the influences of the covalency effect were also neglected. In fact, the $\mathrm{Cu}^{2+}$ centers in $\mathrm{Ca}(\mathrm{OH})_{2}$ may exhibit some covalency and bring forward influences on the SH parameters. Second, the Jahn-Teller clusters $\left[\mathrm{Cu}(\mathrm{OH})_{6}\right]^{4-}$ can experience the Jahn-Teller effect by relaxing the axial (or contracting the perpendicular) $\mathrm{Cu}^{2+}-\mathrm{OH}^{-}$bonds via vibration interactions, which may entirely depress the original trigonal distortion of the host $\mathrm{Ca}^{2+}$ site in pure crystal. Although the Jahn-Teller effect was mentioned in the previous work [13], the contributions from the Jahn-Teller distortions were not quantitatively involved during the calculation procedure, and thus the information of local structures for the impurity centers were not obtained. Third, in the calculations of the hyperfine structure constants, the theoretical $A_{\|}$ are much larger than the observed values and the experimental data of the ${ }^{63} \mathrm{Cu}$ and ${ }^{65} \mathrm{Cu}$ isotopes were not distinguished [13]. As a result, the previous theoretical hyperfine structure constants are not in good agreement with the experimental results. 
Usually, the microscopic mechanisms of the EPR spectra and the information of the two similar but actually different tetragonal $\mathrm{Cu}^{2+}$ centers in $\mathrm{Ca}(\mathrm{OH})_{2}$ can enhance the knowledge of transition-metal ions in crystals and would also be helpful to understand the properties of this material with dopants. In addition, the EPR studies for $\mathrm{Cu}^{2+}$ in original trigonal environments are relatively fewer as compared with the extensive ones in tetragonal or rhombic crystal fields $[10-12,14,15]$. Therefore, further investigations of the $\mathrm{SH}$ parameters and the local structures for $\mathrm{Cu}^{2+}$ in $\mathrm{Ca}(\mathrm{OH})_{2}$ are of fundamental and practical significance.

In this work, the anisotropic $g$ factors and the hyperfine structure constants are theoretically studied for the $\mathrm{Cu}^{2+}$ centers I and II from the high-order perturbation formulae of these parameters for a $3 d^{9}$ ion in tetragonally elongated octahedra. In the calculations, the contributions to the $\mathrm{SH}$ parameters from the covalency effect and the local tetragonal elongation distortions due to the Jahn-Teller effect are quantitatively and uniformly taken into account.

\section{Calculations}

In the lattice of the hexagonal $\mathrm{Ca}(\mathrm{OH})_{2}$, the $\mathrm{Ca}^{2+}$ site is coordinated with six hydroxide ions on the two adjacent layers perpendicular to the $C_{3}$ axis and parallel with the $\mathrm{Ca}^{2+}$ layer [16]. The $\mathrm{Ca}^{2+}$ ion is situated in the center of a slightly trigonally $\left(D_{3 d}\right)$ distorted hydroxide octahedron, with the $\mathrm{Ca}^{2+}{ }_{-} \mathrm{OH}^{-}$bond length $R \approx 2.37 \AA[16]$. When a $\mathrm{Cu}^{2+}$ ion is doped into the lattice of $\mathrm{Ca}(\mathrm{OH})_{2}$, it may locate substitutionally on the $\mathrm{Ca}^{2+}$ site due to the same charge. For a $\mathrm{Cu}^{2+}\left(3 d^{9}\right)$ ion under octahedra, its ground state is the orbital doublet ${ }^{2} E_{\mathrm{g}}$, and the orbital degeneracy cannot be lifted by the trigonal distortion of the hydroxyl octahedron in $\mathrm{Ca}(\mathrm{OH})_{2}$. Nevertheless, the Jahn-Teller ion $\mathrm{Cu}^{2+}$ can suffer the JahnTeller distortion via stretching the $\mathrm{Cu}^{2+}-\mathrm{OH}^{-}$bonds along one direction (i.e., $C_{4}$ axis) and transform the local point symmetry from the original trigonal into tetragonal $\left(D_{4 h}\right)$, which may remove completely the degeneracy of the ground ${ }^{2} E_{\mathrm{g}}$ state. Thus, the impurity $\mathrm{Cu}^{2+}$ actually locates on the tetragonally elongated octahedra in both centers I and II, and the original tiny trigonal distortion of the host $\mathrm{Ca}^{2+}$ site can be entirely depressed. This point is also supported by the studies for $\mathrm{Cu}^{2+}$ on the original trigonally distorted $\mathrm{Cd}^{2+}$ site in $\mathrm{CsCdCl}_{3}$ [17], which actually shows tetragonal elongation distortion due to the Jahn-Teller effect. Thus, the local structures of the centers may be described by the relative elongations $\Delta Z$ along the $C_{4}$ axis due to the Jahn-Teller effect, and the impurity-ligand bond lengths parallel with and perpendicular to the $C_{4}$ axis are expressed as

$$
R_{\|}=R+2 \Delta Z, \quad R_{\perp}=R-\Delta Z .
$$

For a $3 d^{9}\left(\mathrm{Cu}^{2+}\right)$ ion in tetragonally elongated octahedra, the cubic orbital doublet ${ }^{2} E_{\mathrm{g}}$ would be separated into two orbital singlets ${ }^{2} B_{1 \mathrm{~g}}\left(\left|x^{2}-y^{2}\right\rangle\right)$ and ${ }^{2} A_{1 \mathrm{~g}}\left(\left|z^{2}\right\rangle\right)$, with the former lying lowest. Meanwhile, the higher cubic orbital triplet ${ }^{2} T_{2}$ may split into an orbital singlet
${ }^{2} B_{2 \mathrm{~g}}(|x y\rangle)$ and a doublet ${ }^{2} E_{\mathrm{g}}(|x y\rangle,|y z\rangle)$ [15]. Since the covalency of the studied $\mathrm{Ca}(\mathrm{OH})_{2}: \mathrm{Cu}^{2+}$ is not strong and the ligand spin-orbit coupling interaction is very weak, the high order perturbation formulae of the SH parameters based on the conventional crystal-field model [18] containing merely the central ion orbital and spin-orbit coupling contributions can be reasonably adopted here. Thus, we have [18]:

$$
\begin{aligned}
g_{\|} & =g_{\mathrm{s}}+8 k \zeta_{d} / E_{1}+k \zeta_{d}^{2} / E_{2}^{2}+4 k \zeta_{d}^{2} / E_{1} E_{2} \\
& -g_{\mathrm{s}} \zeta_{d}^{2}\left[1 / E_{1}^{2}-1 /\left(2 E_{2}^{2}\right)\right]+k \zeta_{d}^{3}\left(4 / E_{1}-1 / E_{2}\right) / E_{2}^{2} \\
& -2 k \zeta_{d}^{3}\left[2 /\left(E_{1} E_{2}\right)-1 / E_{2}^{2}\right] / E_{1}+g_{\mathrm{s}} \zeta_{d}^{3}\left[1 /\left(E_{1} E_{2}^{2}\right)\right. \\
& \left.-1 /\left(2 E_{2}^{3}\right)\right], \\
g_{\perp} & =g_{\mathrm{s}}+2 k \zeta_{d} / E_{2}-4 k \zeta_{d}^{2} / E_{1} E_{2} \\
& +k \zeta_{d}^{2}\left(2 / E_{1}-1 / E_{2}\right) / E_{2}+2 g_{\mathrm{s}} \zeta_{d}^{2} / E_{1}^{2} \\
& +k \zeta_{d}^{3}\left(2 / E_{1}-1 / E_{2}\right)\left(1 / E_{2}+2 / E_{1}\right) / 2 E_{2} \\
& \left.-g_{\mathrm{s}} \zeta_{d}^{3}\left[1 / E_{1}^{2}-1 /\left(E_{1} E_{2}\right)+1 / E_{2}^{2}\right)\right] /\left(2 E_{2}\right), \\
A_{\|} & =P\left[-\kappa-4 N / 7+\left(g_{\|}-g_{\mathrm{s}}\right)+3\left(g_{\perp}-g_{\mathrm{s}}\right) / 7\right], \\
A_{\perp} & =P\left[-\kappa+2 N / 7-\left(g_{\perp}-g_{\mathrm{s}}\right) / 7\right] .
\end{aligned}
$$

Here $g_{\mathrm{s}}(\approx 2.0023)$ is the spin-only value, $k$ is the orbital reduction factor, $\zeta_{d}$ and $P$ are, respectively, the spin-orbit coupling coefficient and the dipolar hyperfine structure parameter for the $3 d^{9}$ ion in crystals, $\kappa$ is the core polarization constant, $N(\approx k)$ is the average covalency factor, characteristic of the covalency effect of the studied systems, $E_{1}$ and $E_{2}$ are the energy separations between the excited ${ }^{2} B_{2 \mathrm{~g}}$ and ${ }^{2} E_{\mathrm{g}}$ and the ground ${ }^{2} B_{1 \mathrm{~g}}$ states [18], which can be determined from the energy matrices for a $3 d^{9}$ ion under tetragonal symmetry in terms of the cubic field parameter $D_{q}$ and the tetragonal field parameters $D_{s}$ and $D_{t}$ :

$$
\begin{aligned}
& E_{1} \approx 10 D_{q}, \\
& E_{2} \approx 10 D_{q}-3 D_{s}+5 D_{t} .
\end{aligned}
$$

The tetragonal field parameters are usually calculated from the local geometrical relationship of the studied impurity centers using the superposition model [19]:

$$
\begin{aligned}
& D_{s} \approx(4 / 7) \bar{A}_{2}(R)\left[\left(R / R_{\|}\right)^{t 2}-\left(R / R_{\perp}\right)^{t 2}\right], \\
& D_{t} \approx(32 / 21) \bar{A}_{4}(R)\left[\left(R / R_{\|}\right)^{t 4}-\left(R / R_{\perp}\right)^{t 4}\right] .
\end{aligned}
$$

Here $t_{2} \approx 3$ and $t_{4} \approx 5[19]$ are the power-law exponents due to the dominant ionic nature of the bonds. $\bar{A}_{2}(R)$ and $\bar{A}_{4}(R)$ are the intrinsic parameters, with the reference distance $R$. For octahedral $3 d^{n}$ clusters, the relationships $\bar{A}_{4}(R) \approx(3 / 4) D_{q}[19]$ and $\bar{A}_{2}(R) \approx 10.8 \bar{A}_{4}(R)$ [20-22] have been proved valid in many crystals and are reasonably applied here. From the above expressions, the SH parameters (especially the anisotropy $\Delta g=g_{\|}-g_{\perp}$ ) are correlated to the tetragonal field parameters and hence to the local structures of the impurity centers. 
Since no optical spectra were reported for $\mathrm{Ca}(\mathrm{OH})_{2}: \mathrm{Cu}^{2+}$, the spectral parameters $D_{q} \approx$ $1250 \mathrm{~cm}^{-1}$ and $N \approx 0.88[23]$ for the $\left[\mathrm{Cu}\left(\mathrm{H}_{2} \mathrm{O}\right)_{6}\right]^{2+}$ clusters in hydrates can be reasonably adopted here. Then the spin-orbit coupling coefficient $\zeta_{d}$ for $\mathrm{Cu}^{2+}$ in $\mathrm{Ca}(\mathrm{OH})_{2}$ is obtained from the free-ion value $\zeta_{d}^{0}$ $\left(\approx 829 \mathrm{~cm}^{-1}\right.$ [24]) multiplying $N$. In the formulae of the hyperfine structure constants, the dipolar hyperfine structure parameter $P \approx 388 \times 10^{-4}$ and $416 \times 10^{-4} \mathrm{~cm}^{-1}$ for ${ }^{63} \mathrm{Cu}$ and ${ }^{65} \mathrm{Cu}$, respectively [25].
As for the core polarization constant, the expectation value is about 0.3 [24] for $3 d^{n}$ ions in crystals, and those $(\approx 0.26-0.32[26])$ for $\mathrm{Cu}^{2+}$ in tutton salts were also reported. Since the magnitude of $A_{\|}$depends largely upon the isotropic term proportional to $\kappa$ (see Eq. (2)), the slightly $(6 \%)$ higher $A_{\|}$for center II than for center I should be ascribed to the slightly larger $\kappa$ of the former. Thus, one can reasonably estimate $\kappa \approx 0.3$ and 0.32 for centers I and II, respectively.

The anisotropic $g$ factors and the hyperfine structure constants (in $10^{-4} \mathrm{~cm}^{-1}$ )

TABLE for the two tetragonal $\mathrm{Cu}^{2+}$ centers in $\mathrm{Ca}(\mathrm{OH})_{2}$.

\begin{tabular}{c|c|c|c|c|c|c|c}
\hline \hline & & $g_{\|}$ & $g_{\perp}$ & ${ }^{63} A_{\|}$ & ${ }^{65} A_{\|}$ & ${ }^{63} A_{\perp}$ & ${ }^{65} A_{\perp}$ \\
\hline \multirow{4}{*}{ Center I } & Cal. $^{a}$ & 2.421 & 2.079 & \multicolumn{2}{|c|}{-195.2} & \multicolumn{2}{|c}{5.8} \\
\cline { 2 - 8 } & Cal. $^{b}$ & 2.418 & 2.077 & -122.8 & -131.7 & -3.9 & -4.2 \\
\cline { 2 - 8 } & Exp. [13] & $2.421(1)$ & $2.079(1)$ & $-123.3(1)$ & $-132.6(1)$ & $<10$ & $<10$ \\
\hline \multirow{4}{*}{ Center II } & Cal. $^{a}$ & 2.408 & 2.080 & \multicolumn{2}{|c|}{-176.7} & \multicolumn{2}{|c}{-0.3} \\
\cline { 2 - 8 } & Cal. $^{b}$ & 2.405 & 2.080 & -129.3 & -138.6 & -3.0 & -3.2 \\
\cline { 2 - 8 } & Exp. [13] & $2.401(1)$ & $2.081(1)$ & $-130.7(1)$ & $-141.0(1)$ & $<10$ & $<10$ \\
\hline
\end{tabular}

a Calculations based on the simple second-order perturbation formulae and the various adjustable parameters in the previous work [13].

${ }^{b}$ Calculations based on the high order perturbation formulae and the tetragonal elongations $\Delta Z$ due to the Jahn-Teller effect in this work.

In the formulae of the SH parameters, only the tetragonal elongation $\Delta Z$ is unknown. Substituting these values into Eq. (2) and fitting the calculated SH parameters (especially the anisotropy $\Delta g$ ) to the experimental data, we have

$$
\Delta Z \approx 0.083 \text { and } 0.065 \AA
$$

for centers I and II, respectively. The corresponding results $\left(\right.$ Cal $\left.^{b}\right)$ are shown in Table. For comparisons, the calculation results $\left(\mathrm{Cal}^{a}{ }^{a}\right.$ ) based on the simple second-order perturbation formulae and the various adjustable parameters (i.e., the spin-orbit coupling constants $u, w$ and the core polarization constant $\kappa)$ in the previous work [13] are also listed in Table.

\section{Discussion}

Table reveals that the calculated $\mathrm{SH}$ parameters (especially $A_{\|}$in $\mathrm{Cal}^{b}{ }^{b}$ ) for both centers based on the tetragonal elongations of the Jahn-Teller nature in Eq. (5) show better agreement with the observed values than those $\left(\mathrm{Cal}^{a}{ }^{a}\right.$ ) based on the simple second-order perturbation formulae and various adjustable parameters in the previous work [13]. Thus, the EPR spectra for $\mathrm{Cu}^{2+}$ in $\mathrm{Ca}(\mathrm{OH})_{2}$ are uniformly and satisfactorily interpreted, and the information about local structures of the impurity centers is also obtained.

1) The positive signs of the tetragonal elongations $\Delta Z$ are in accordance with the expectation based on the Jahn-Teller effect, corresponding to the positive anisotropy $\Delta g$ (see Eq. (1)). Meanwhile, the small values of $\Delta Z(\approx 0.085$ and $0.063 \AA)$ are consistent with the slight anisotropies $\Delta g(\approx 0.342$ and 0.320$)$ for centers I and II, respectively. Interestingly, the relative elongation ratio $\Delta Z / R(\approx 3-4 \%)$ for $\mathrm{Cu}^{2+}$ in $\mathrm{Ca}(\mathrm{OH})_{2}$ in this work is also comparable with that $(\approx 3 \%[27])$ for $\mathrm{Cu}^{2+}$ on the octahedral interstitial site in the CdSe nanocrystals based on the EPR analysis and can be regarded as reasonable. Further, the higher $\Delta Z$ for center I than that for center I agrees well with the larger tetragonal distortion and hence the larger $\Delta g$ of the former. In fact, the larger Jahn-Teller elongation for center I can be stabilized at low temperature $(87 \mathrm{~K})$, whereas the relatively smaller $\Delta Z$ for center II may be ascribed to the depression in the elongation due to the obvious thermal fluctuation (averaging effect) at room temperature. Therefore, the original trigonal distortion of the host $\mathrm{Ca}^{2+}$ site in $\mathrm{Ca}(\mathrm{OH})_{2}$ is entirely depressed in the impurity centers. Similar tetragonal elongations of the ligand octahedra were reported for $\mathrm{Cu}^{2+}$ on the trigonal $\mathrm{Cd}^{2+}$ site in $\mathrm{CsCdCl}_{3}$ and $\mathrm{Mg}^{2+}$ site in lanthanum magnesium nitrate [28] due to the Jahn-Teller effect.

2) As for the calculated hyperfine structure constants $\left(\mathrm{Cal}^{a}{ }^{a}\right)$ based on the various adjustable parameters in the previous work [13], the $A_{\|}$are much (about 30-50\%) larger than the observed values, and the experimental data were not distinguished for the ${ }^{63} \mathrm{Cu}$ and ${ }^{65} \mathrm{Cu}$ 
isotopes. This can be ascribed to the simple $A$ formulae and failing to connect the EPR analysis with the local structures of the impurity centers. According to Eq. (2), the hyperfine structure constants depend sensitively upon the isotropic terms proportional to the core polarization constant $\kappa$. In fact, $\kappa$ is the characteristic of the Fermi contact interactions (or admixtures) between the ground $3 s^{2} 3 d^{9}$ configuration and the excited $s$-orbital (e.g. $3 s 3 d^{9} 4 s$ ) states of the central ion in crystals [15]. For the lowest ${ }^{2} B_{1}\left(\left|x^{2}-y^{2}\right\rangle\right)$ state of $\mathrm{Cu}^{2+}$ in the elongated octahedra, the higher $\Delta Z$ of center I at $87 \mathrm{~K}$ may lead to less significant $\mathrm{Cu}^{2+} 3 d-3 s$ (or $-4 s$ ) admixtures and hence the lower $\kappa$. Thus, the slightly lower magnitudes $\kappa(\approx 0.30)$ and $A_{\|}$ $\left(\approx(-123--133) \times 10^{-4} \mathrm{~cm}^{-1}\right)$ for center I than those $\left(\kappa \approx 0.32\right.$ and $\left.A_{\|} \approx(-131--141) \times 10^{-4} \mathrm{~cm}^{-1}\right)$ for center II can be understood. Moreover, the large magnitudes of $A_{\|}$for both centers are understandable in view of the isotropic term enhanced by the anisotropic one of the same negative sign, while the tiny $A_{\perp}$ can be ascribed to the fact that the isotropic and anisotropic terms almost cancel one another. In addition, the signs of the hyperfine structure constants were not experimentally determined for $\mathrm{Ca}(\mathrm{OH})_{2}: \mathrm{Cu}^{2+}$ in Ref. [13]. Based on the present calculations and the experimental data for $\mathrm{Cu}^{2+}$ in various tetragonally elongated octahedra [25], both signs are found to be negative.

3) There may be some errors in the present calculations. Firstly, the approximations of the theoretical model and formulae can induce some errors in this work. Secondly, the conventional formulae of the SH parameters are adopted in the theoretical treatments, while the contributions from the ligand orbital and spin-orbit coupling interactions are not taken into account. Fortunately, the studied $\mathrm{Cu}^{2+}$ centers in $\mathrm{Ca}(\mathrm{OH})_{2}$ exhibit only weak covalency (characterized by $N \approx 0.9$ ), and the spin-orbit coupling coefficient $\left(\approx 151 \mathrm{~cm}^{-1}[29]\right)$ of $\mathrm{O}^{2-}$ is much smaller than that $\left(\approx 829 \mathrm{~cm}^{-1}[24]\right)$ of $\mathrm{Cu}^{2+}$. The above influences due to the omission of the ligand contributions can be regarded as very small and negligible. Thirdly, the errors also arise from the approximation of the relationship $\bar{A}_{2}(R) \approx 10.8 \bar{A}_{4}(R)$, which would bring forward influences on the tetragonal field parameters and the final SH parameters. The errors of $\Delta Z$ are estimated to be no more than $0.7 \%$ as the ratio $\bar{A}_{2}(R) / \bar{A}_{4}(R)$ varies by $10 \%$.

\section{Conclusion}

The SH parameters and the local structures for the two tetragonal $\mathrm{Cu}^{2+}$ centers in $\mathrm{Ca}(\mathrm{OH})_{2}$ are theoretically studied from the high order perturbation formulae of a tetragonally elongated octahedral $3 d^{9}$ cluster. The ligand octahedra around $\mathrm{Cu}^{2+}$ are found to suffer the relative elongations of about $0.083 \AA$ and $0.065 \AA$ for centers I and II, respectively, along the $C_{4}$ axis due to the Jahn-Teller effect, which may entirely depress the original slight trigonal distortion of the host $\mathrm{Ca}^{2+}$ site.
The theoretical results (particularly the hyperfine structure constants) of this work show some improvements as compared to those of the previous work.

\section{Acknowledgments}

This work was financially supported by the Fundamental Research Funds for the Central Universities under grant No. ZYGX2010J047.

\section{References}

[1] P. Baranek, A. Lichanot, R. Orlando, R. Dovesi, Chem. Phys. Lett. 340, 362 (2001).

[2] R.A. Buchanan, H.H. Caspers, J. Murphy, Appl. Opt. 2, 1147 (1963).

[3] T. Nagai, T. Ito, T. Hattori, T. Yamanaka, Phys. Chem. Miner. 27, 462 (2000).

[4] Y. Syono, M.H. Manghnani, High-Pressure Research: Application to Earth and Planetary Sciences, Terra Scientific Publ. Co. American Geophysical Union, New York 2005.

[5] A. Martinez, M.E. Rodriguez, S.W. York, J.F. Preston, L.O. Ingram, Biotechnol. Bioeng. 69, 526 (2000).

[6] B.P.F. de A. Gomes, C.C.R. Ferraz, M.E. Vianna, P.L. Rosalen, A.A. Zaia, F.B. Eixeira, F.J. De Souza-Filho, Braz. Dent. J. 13, 155 (2002).

[7] F. Holuj, C.T. Kwan, J. Magn. Reson. 18, 123 (1975).

[8] R.S. de Biasi, M.L.N. Grillo, J. Phys. Chem. Solids 60, 1905 (1999).

[9] F. Holuj, C.T. Kwan, J. Magn. Reson. 17, 381 (1975).

[10] Z. Sroubek, K. Zdansky, J. Chem. Phys. 44, 3078 (1965).

[11] R.W. Reynolds, L.A. Boatner, M.M. Abraham, Y. Chen, Phys. Rev. B 10, 3802 (1974).

[12] K.V. Narasimhulu, C.S. Sunandana, J.L. Rao, J. Phys. Chem. Solids 61, 1209 (2000).

[13] R.G. Wilson, F. Holuj, N.E. Hedgecock, Phys. Rev. B 1, 3609 (1970).

[14] T. Kobayashi, H. Katsuda, K. Hayashi, M. Tokumoto, H. Ihara, Jpn. J. Appl. Phys. 27, L670 (1988).

[15] A. Abragam, B. Bleaney, Electron Paramagnetic Resonance of Transition Ions, Oxford University Press, London 1970

[16] H.E. Petch, Acta Crystallogr. 14, 950 (1961).

[17] S.O. Graham, R.L. White, Phys. Rev. B 10, 4505 (1974).

[18] W.H. Wei, S.Y. Wu, H.N. Dong, Z. Naturforsch. A 60, 541 (2005).

[19] D.J. Newman, B. Ng, Rep. Prog. Phys. 52, 699 (1989).

[20] W.L. Yu, X.M. Zhang, L.X. Yang, B.Q. Zen, Phys. Rev. $B$ 50, 6756 (1994).

[21] D.J. Newman, D.C. Price, W.A. Runciman, Am. Miner. 63, 1278 (1978).

[22] Z.Y. Yang, J. Phys. Condens. Matter. 12, 4091 (2000). 
[23] A.S. Chakravarty, Introduction to the Magnetic Properties of Solids, Wiley-Interscience Publication, New York 1980.

[24] J.S. Griffith, The Theory of Transition-Metal Ions, Cambridge University Press, London 1964.

[25] B.R. McGarvey, J. Phys. Chem. 71, 51 (1967).

[26] A. Abragam, M.H.I. Pryce, Proc. R. Soc. (Lond.) A 206, 164 (1951)
[27] S.Y. Wu, H.M. Zhang, P. Xu, S.X. Zhang, Spectrochim. Acta A 75, 230 (2010).

[28] D.P. Breen, D.C. Krupka, F.I.B. Williams, Phys. Rev. 179, 241 (1969).

[29] E.K. Hodgson, I. Fridovich, Biochem. Biophys. Res. Commun. 54, 270 (1973). 